\title{
Demographic, Behavioural and Normative Risk Factors for Gambling Problems Amongst Sports Bettors
}

\author{
Nerilee Hing $^{1}$ - Alex M. T. Russell ${ }^{1}$ - Peter Vitartas ${ }^{2}$ • \\ Matthew Lamont ${ }^{1}$
}

Published online: 5 September 2015

(C) Springer Science+Business Media New York 2015

\begin{abstract}
Sports betting is growing exponentially, is heavily marketed and successfully targets young adult males. Associated gambling problems are increasing. Therefore, understanding risk factors for problem gambling amongst sports bettors is an increasingly important area of research to inform the appropriate design and targeting of public health and treatment interventions. This study aimed to identify demographic, behavioural and normative risk factors for gambling problems amongst sports bettors. An online survey of 639 Australian sports bettors using online, telephone and retail betting channels was conducted. Results indicated that vulnerable sports bettors for higher risk gambling are those who are young, male, single, educated, and employed full-time or a full-time student. Risk of problem gambling was also found to increase with greater frequency and expenditure on sports betting, greater diversity of gambling involvement, and with more impulsive responses to betting opportunities, including in-play live action betting. Normative influences from media advertising and from significant others were also associated with greater problem gambling risk. The results of this study can inform a suite of intervention, protection and treatment initiatives targeted especially at young male adults and adolescents that can help to limit the harm from this gambling form.
\end{abstract}

Nerilee Hing

nerilee.hing@scu.edu.au

Alex M. T. Russell

alex.russell@scu.edu.au

Peter Vitartas

p.vitartas@latrobe.edu.au

Matthew Lamont

matthew.lamont@scu.edu.au

1 Centre for Gambling Education and Research, Southern Cross University, PO Box 157, Lismore, NSW, Australia

2 La Trobe Business School, Latrobe University, Bundoora, VIC 3086, Australia 
Keywords Sports betting - Risk factors - Problem gambling - Young males · Interventions

\section{Introduction}

Sports betting refers to wagering on approved types of local, national or international sporting activities (other than horse and greyhound racing), whether on- or off-course, in person, by telephone or via the internet (QGSO 2014). Sports betting comprises a major sector of the gambling industry, generating about one-seventh of gambling industry profits worldwide (GBGC 2013). Significant future growth is expected; annual gross profits (bettor losses) from regulated sports betting are forecast to increase from US $\$ 58$ billion in 2012 to US $\$ 70$ billion by 2016 , with the unregulated sports betting sector understood to be many times larger (Foley-Train 2014). Internet and mobile technologies have been key drivers of growth, enabling expansion of services across national borders by multinational corporate bookmakers offering online sports betting services, while providing easy access, convenience, anonymity and better prices for consumers (Gainsbury et al. 2012; Hing et al. 2014a, b, c). In most jurisdictions, sports wagers can also be placed by telephone and in physical retail outlets. As in many countries, sports betting via the Internet is growing in popularity, at the expense of betting via telephone and retail outlets. For example, estimates in 2013 were that $40 \%$ of Australian wagering (including both sport and races) took place online, $40-45 \%$ at off-course agencies, and only a small proportion by telephone (JSCGR 2013), with the online proportion for sports betting likely to have increased since then.

Several researchers have argued that the growth of sports betting is increasingly contributing to the incidence of problem gambling (Hing et al. 2014b, c; Lamont et al. 2011; McMullan 2011; Sproston et al. 2015). A comprehensive worldwide review of gambling prevalence studies conducted between 1975 and 2012 identified 18 national studies for various countries, along with 15 US, three Australian and two Canadian provincial studies where sports betting was one of the gambling forms most strongly associated with problem gambling (Williams et al. 2012). Multivariate analyses of large nationally representative US surveys have also found sports betting participation to be a predictor of problem gambling, when controlling for engagement in other forms of gambling (Kessler et al. 2008; Welte et al. 2004). Studies of Internet gamblers have linked sports betting to problem gambling. For example, amongst Internet gamblers in a weighted sample of 4688 Australian gamblers, $16 \%$ identified sports betting as their most problematic gambling form (Gainsbury et al. 2013). Evidence from treatment services also indicates that gambling problems amongst sports bettors are increasing. One Australian clinic has noted a rapid rise in reported problems with sports betting, increasing from $<5 \%$ of clients in 2006-2007 to 15-20 \% of new clients in 2010-2011 (University of Sydney Gambling Treatment Clinic 2011). Similarly, sports betting was the most commonly reported principal gambling activity for $40 \%$ of Internet gamblers attending NSW gambling help services in 2011-2012, increasing from $25 \%$ in 2007-2008 (Hing et al. 2014b).

Despite these indications that sports betting may be increasingly contributing to problem gambling, no research appears to have comprehensively examined risk factors for problem gambling amongst sports bettors. This study begins to address this gap. More specifically, it aimed to identify demographic, behavioural and normative risk factors for gambling problems amongst sports bettors. Identifying these risk factors can usefully 
inform appropriate design and targeting of public health and treatment interventions. This is important, given that most interventions currently focus on electronic gaming machine (EGM) gamblers. The growth of sports betting and related problems suggests that interventions may also need to be tailored to this emerging group of high risk gamblers.

\section{Literature Review}

\section{Demographic Risk Factors}

Access to wagering via Internet and mobile platforms has broadened the customer base and created opportunities for wagering operators to access previously untapped demographic groups (Foley-Train 2014). In contrast to previous profiles of bettors as predominantly older working class men, numerous recent prevalence surveys have found that contemporary sports bettors tend to be young adult males aged 18-34 years (Humphreys and Perez 2012; Sproston et al. 2012; Wardle and Seabury 2012). Two studies with large international samples found that over $90 \%$ of online sports bettors were male (LaBrie et al. 2007; Wood and Williams 2011), with an average age of 31 years (LaBrie et al. 2007).

Studies and reviews have consistently found that being a young adult male is a risk factor for problem gambling (Delfabbro 2012; Hing et al. 2015b; Johansson et al. 2009; Williams et al. 2012; Williams et al. 2012), including amongst Internet gamblers. For example, risk factors for greater problem gambling severity amongst a weighted sample of 3239 Australian Internet gamblers included being male, younger, and a non-English speaker at home, as well as greater gambling participation (Hing et al. 2014b). Concern has been raised that young adult men who have embraced sports betting are at heightened risk of related gambling problems, particularly given their pre-existing vulnerability to problem gambling (Lamont et al. 2011; McMullan 2011; Thomas et al. 2012). Hing et al.'s study (2014b) also found that betting on sports, races and poker was a risk factor for greater problem gambling severity amongst Australian Internet gamblers. Treatment services also report that young men in particular are increasingly reporting difficulties in controlling their online sports betting (Blaszczynski and Hunt 2011).

Overall however, empirical evidence on demographic factors associated with high and low levels of problem gambling is thin. US studies of sports bettors have been restricted to account-holders with only a single online wagering operator (e.g., Gray et al. 2012; LaBrie et al. 2007; LaBrie and Shaffer 2011; LaPlante et al. 2014; Xuan and Shaffer 2009), while studies of Internet gamblers have not analysed demographic factors specifically for high and low risk sports bettors (e.g., Hing et al. 2014b; Wood and Williams 2011). Australian surveys of sports bettors have also not ascertained demographic risk or protective factors (Sproston et al. 2015). The current study investigates a range of demographic characteristics of sports bettors to identify those associated with higher problem gambling severity.

\section{Behavioural Risk Factors}

Specific features of Internet gambling have been reported as contributing to impaired control by moderate risk/problem gamblers, including sports bettors. These include use of digital money, access to credit, lack of scrutiny, easy 24/7 accessibility, fast speed and wide range of betting opportunities, and tempting promotional offers (Hing et al. 2014a). 
While these features are common to the betting experience for all online sports bettors, little is known about specific betting behaviours that characterise those with gambling problems.

Insights into behavioural factors associated with highly involved sports betting are provided by studies examining actual betting data, although these have been drawn only from single online operators. These studies have found that overall betting levels are moderate, and that bettors adjust their betting frequency, number of bets and stake size based on wins and losses, suggesting that online sports betting is not inherently associated with excessive gambling (LaBrie et al. 2007; LaPlante et al. 2008). However, a group of heavily involved bettors has been identified, whose adaptation is slower or absent, and who maintain high involvement in live action betting (in-play betting on short-term in-match contingencies) (LaBrie et al. 2007; LaPlante et al. 2008). Other studies have found that bettors who exceed operator imposed deposit limits, utilise the site's self-limiting facilities, or trigger a responsible gambling alert system place more bets than the rest of the sample prior to using these interventions (Broda et al. 2008; Gray et al. 2012; Nelson et al. 2008). However, a measure of problem gambling was not included in any of the above studies, so whether these betting behaviours are associated with gambling problems cannot be determined. Two analyses have been based on self-reported gambling problems. Xuan and Shaffer (2009) compared betting data of 226 bettors who closed their account because of self-reported gambling problems, to a matched sample not closing their account. Leading up to account closure, the former group experienced increasing monetary losses and increased their stake per bet, but were more likely to choose bets with shorter odds compared to controls. LaBrie and Shaffer (2011) compared problem gambling account closers to those who had closed accounts for other reasons. About half of the former exhibited a distinct homogeneous behavioural pattern of more and larger bets, more frequent betting, and intense betting soon after opening their betting account.

The above studies help to characterise the betting behaviour of highly involved sports bettors and those self-reporting gambling problems. However, attempts to determine whether this high-risk group comprises a qualitatively distinctive category of gamblers were unsuccessful; instead, their behaviours were found to lie at the extreme of the dimensions underlying the behaviours of recreational sports bettors (Braverman et al. 2011). Perhaps restriction of data to one online betting site obscures results or, as argued by Philander (2014), bet intensity, variability, frequency and trajectory are insufficient variables to accurately classify probable problem gamblers. A subsequent study using operator data included a gambling disorder screen completed by 1440 subscribers (LaPlante et al. 2014). Participation in live action online sports betting was the only gambling form significantly associated with potential gambling-related problems, when controlling for involvement in another 15 gambling activities. The number of days an individual gambled was also statistically significant.

Overall, the above studies suggest that numerous, frequent and larger bets appear likely to distinguish high-risk sports bettors from their lower risk counterparts. Also implicated was in-play betting on live action events (Braverman et al. 2013; Gray et al. 2012; LaPlante et al. 2008, 2014; Nelson et al. 2008). These types of bets were found to be particularly attractive to problem gamblers in a study measuring sports bettors' responses to different message elements in sports betting promotions (Hing et al. 2014c). Live action betting may be inherently risky as it provides opportunities for fast-paced continuous betting, and requires quick and perhaps impulsive decisions without much time for reflection (Hing et al. 2014a, b, c; Nelson et al. 2008). The current study assesses whether specific betting behaviours, including participation in live action betting, impulse betting, 
and greater sports betting frequency and expenditure, are associated with gambling problems amongst sports bettors.

\section{Normative Risk Factors-Media and Family/Friends}

The media and significant others are two key socialising agents that can act to normalise certain attitudes and behaviors associated with gambling. Proliferation of sports betting advertising is a major industry trend (GBGC 2013) and sports betting operators are increasingly prominent amongst the plethora of brands paying for sponsorship and media rights which now make up over half of global sports sector revenues (PWC 2011). Several studies have documented the growing prominence of televised, online and social media sports betting promotion (ACMA 2013; Gainsbury et al. 2015; Hing et al. 2014a; McMullen 2011; Sproston et al. 2015). Sports betting operators use a range of marketing strategies to recruit, register and retain online account-holders. A content analysis of 40 large online wagering, poker and casino sites (Weibe 2008) identified examples of recruitment strategies as advertising on search engines and affiliate networks, traditional advertising through television, radio and print, pop-ups and banner displays on websites, event or team sponsorships and creative guerilla marketing tactics. Sign-up, refer-a-friend and welcome bonuses are examples of registration strategies, while bonus and rewards programs, such as loyalty programs, can help to retain customers (Weibe 2008).

The increased integration of sports betting logos, signage and promotions within televised sports broadcasts and at live sporting fixtures has raised particular concerns that sports betting is becoming normalised amongst sports audiences (Lamont et al. 2011; Sproston et al. 2015; Thomas et al. 2012). This marrying of sports and sports betting cultures has led to the 'gamblification of sport' and the 'sportification of gambling' (McMullen 2011, p. 4), where sports fans are encouraged to gamble as an extension of their interest in sport (Welte et al. 2004), and betting is promoted as a healthy harmless activity akin to playing sport (Lamont et al. 2011). There may be important complementarities in watching sports and betting on sports (Humphreys and Perez 2012), with a key market for both activities being young adult males (Borland and Macdonald 2003; Hammervold and Solberg 2006; Milner et al. 2013).

Growing normalisation of sports betting has been attributed to this enhanced promotion. Participants in Sproston et al.'s study (2015) described how regular discussions and placements of bets now occur in social and workplace settings, with advertisements portraying gambling as an everyday activity, an important social lubricant amongst young adult males, and an activity no longer associated with any social stigma. Respondents also noted that sports betting promotion has increased how much their friends and family talk about sports betting, and their significant others' interest in sports betting, desire to bet on sports and actual sports betting behaviour. Similarly, Thomas et al. (2012) found a growing sub-culture of sports betting amongst young adult males who reported peer group pressure to bet in order to fit in with their friends. Gordon et al. (2015) researched lifestyle consumption communities amongst 18-30 year old sports bettors in Australia. They found that sports betting was embedded in their everyday lives, regularly featured in their leisure and social interactions, manifest in their sub-culture and was highly normalised. Shared cultural values within their group, within-group rivalry, loyalty to favoured sports teams, and the desire to display betting acumen, skills and knowledge drove some risky consumption practices that could provide a pathway to sports betting problems.

Previous research has found that problem and at-risk gamblers report greater media exposure to sports betting advertising and promotions and higher agreement that this has 
increased positive social norms of family and friends in relation to sports betting (Hing et al. 2015a; Sproston et al. 2015). The current study examines whether the normative effects of both the media and significant others are associated with gambling problems amongst sports bettors.

Overall, this study aimed to identify demographic, behavioural and normative risk factors for gambling problems amongst sports bettors. As explained further below, demographic factors investigated comprised age, gender, marital status, household type, highest educational qualification, work status, household income, and country of birth. Behavioural factors included frequency and expenditure on sports betting and a range of sports betting behaviours. Normative factors comprised exposure to sports betting advertising and promotions, and normative influences on sports betting from significant others.

\section{Methods}

\section{Sampling}

A university human research ethics committee granted approval for this project. Because only $13 \%$ of the Australian adult population gambles on sport (Hing et al. 2014b), gaining a random sample of sports bettors was not feasible. Instead, a purposive sample was recruited through a market research company comprising 639 adults in Queensland Australia who had bet on sports in the previous 12 months. Queensland was selected as the jurisdiction of interest because the study was funded by a Queensland Government grants scheme. 'At least fortnightly' sports bettors were deliberately oversampled to optimise recruitment of adequate numbers of problem and at-risk gamblers to examine risk factors for gambling problems. The survey was conducted online between October and December 2012 by a reputable online panel survey company who maintained control for respondent quality through respondent completion times and attention filters.

\section{Measures}

\section{Outcome Variable}

Total score on the Problem Gambling Severity Index (PGSI; Ferris and Wynne 2001) was the outcome variable. Its nine items are scored as never $=0$, sometimes $=1$, most of the time $=2$ and almost always $=3$. Total scores range from 0 to 27 . The PGSI is widely used in many countries, including Australia, and is a recommended measure of problem gambling severity (Problem Gambling Research and Treatment Centre 2011).

\section{Demographic Variables}

Age, gender, marital status, household type, highest educational qualification, work status, household income, and country of birth were ascertained.

Age was measured in age categories: 18-24 years, then in 10-year categories up to $65+$ years. Household type response options were: single person, one parent family with children, couple with children, couple with no children, group household, or other. Work status response options were: work full-time, work part-time, self-employed, unemployed, 
full-time student, full-time home duties, retired, sick or disability pension, and other. Respondents could select just one response.

Some variables were recoded to avoid small cell sizes. The eight respondents who were widowed were added to the 'divorced or separated' category. Thus, there were four categories for marital status: married, living with partner/de facto, divorced/separated/widowed and never married. Similarly, 11 respondents reported that their highest level of education was completing primary school and two did not complete primary school. These respondents were added to the 80 'Year 10 or equivalent' respondents, leaving five levels of education: postgraduate qualifications, undergraduate university or college degree, trade/technical certificate or diploma, Year 12 or equivalent, and less than Year 12. Country of birth was recoded as 'Australia' or 'other'.

\section{Gambling Behaviour Variables}

Frequency of sports betting during the previous 12 months was ascertained on a sevenpoint scale from never to daily for each of up to 12 sports (rugby league, Australian Rules Football, rugby union, soccer, cricket, motor racing, golf, tennis and up to four "Other" sports, in which the respondent was asked to state which sport they had bet on and how often). These 12 frequency variables displayed good reliability (Cronbach's alpha $=0.95$ ) and a single score for sports betting frequency was calculated, with higher scores indicating more frequent betting. Past year sports betting expenditure was measured as AU\$ per month or per year (converted into annual expenditure). Respondents were also asked how many sports betting agencies they held an account with during the last 12 months.

Five questions about sports betting behaviours during the past 12 months each required percentage allocations amongst response options to total $100 \%$ :

- channels used to place sports bets; response options were Internet, telephone (not using the Internet), and land-based venues;

- timing of sports bets; response options were before the day of the match they were betting on, on the day of that match and at least an hour before match commencement, within the hour immediately before match commencement, and during the match they were betting on;

- percentage of sports bets that were 'researched and planned in advance of the match', 'on impulse before the start of the match', and 'on impulse during the match';

- percentage of sports bets placed before match commencement that were on the final outcome of the match and on key events within the match; and

- percentage of sports bet placed during a match that were on 'the final outcome of the match', 'key events within the match (exotic bets) e.g., who will score the next goal', and 'micro events within the match (micro bets), e.g., the outcome of the next ball in cricket or the next point in tennis'.

The number of other gambling forms engaged in during the last 12 months was derived by asking frequency of participation in another seven forms of gambling that are readily available in Queensland (EGMs, lottery/lotto or scratch lottery tickets, race betting, table games at a land-based casino, casino games on the Internet, poker tournaments, keno).

\section{Normative Variables}

Televised and live sporting events are media through which Australians gain most exposure to sports betting advertising and promotions (Sproston et al. 2015), so exposure to 
these events was deemed an appropriate proxy measure of media influences on sports betting. Respondents were asked frequency of watching sporting events on television and live at the ground for eight different sports where sports betting is most prominently promoted in Australia (rugby league, Australian Rules Football, rugby union, soccer, cricket, tennis, golf, motor sports). Frequency of watching was measured on a seven-point scale, from never to daily.

Normative influences on sports betting from significant others was measured with an adapted version of the Subjective Norms: Family and Friends Scale (Moore and Ohtsuka 1997) which asked how strongly the respondent agrees or disagrees on a five-point Likert scale with 12 statements about how their family and friends feel about gambling (e.g., 'most of my friends approve of gambling', 'people in my family often go to places where gambling occurs'). These questions were adapted to specifically refer to sports betting rather than to gambling in general. After appropriate item reversals, family items and friend items were summed and then multiplied by the respective statement assessing motivation to comply with those norms. Both subscales were then summed to create a single measure (Cronbach's alpha $=0.72$ ). Higher scores on this scale indicate more positive attitudes towards sports betting. Additionally, all results that were significant for the overall subjective norms scale were also significant for each of the family and friends subscales. The subscales were correlated $(r=0.602)$ and highly correlated with the overall scale ( $r=0.837$ and 0.929$)$. Thus, we opted to report the results from the overall scale rather than the subscales, as the results were essentially identical for the subscales.

\section{Analysis}

The dependent variable for all analyses was total score on the PGSI. PGSI scores can be used to group respondents into four categories of risk, but raw scores were considered more appropriate here as they better capture differences in problem gambling severity. For example, individuals scoring 8-27 are classified as problem gamblers, yet clearly experience different levels of problem gambling symptoms. The median score on the PGSI was 1 and $49.8 \%$ of the sample had a score of 0 , with the remaining scores ranging all the way to 27 in a distribution that was positively skewed (skewness $=1.76$ ).

As scores on the PGSI were highly skewed, non-parametric statistics were conducted. These tests include Spearman's correlations for ordinal, interval or ratio independent variables and Kruskal-Wallis and Mann-Whitney $U$ tests for nominal independent variables. Where the Kruskal-Wallis test was used, post hoc pairwise tests were conducted using a series of Mann-Whitney $U$ tests. Mean ranks are reported along with medians. We note that the mean rank for a particular group on any variable changes depending on who that groups is being compared to, which is why (for example) never married respondents are reported with a mean rank of 245.88 when compared to married respondents, but a mean rank of 139.71 when compared to respondents living with their partner/de facto. All analyses were conducted using an alpha of 0.05 unless stated otherwise.

Given that the purpose of the study was to identify risk factors, multivariate analyses were not conducted as these may obscure identification of distinctive characteristics associated with high risk sports betting that can inform the targeting of treatment and public health interventions (even though they may not be causal factors). 


\section{Results}

\section{Demographics}

The 408 males in the sample had significantly higher PGSI scores (median $=1$, mean rank $=335.98$ ) compared to the 231 females (median $=0$, mean rank $=291.78$ ), MannWhitney $U=40,605.5, Z=-3.11, p=0.002$. Younger respondents were significantly more likely to have higher PGSI scores compared to older respondents, Spearman's rho $=-0.31, p<0.001$. No significant difference was found in PGSI scores between those who were born in Australia and elsewhere, Mann-Whitney $U=30,004.5$, $Z=-.155, p=0.122$.

Significant differences were found between the different marital statuses, KruskalWallis $\chi(3)=9.83, p=0.020$. Post-hoc tests revealed that the 133 never married respondents had significantly higher PGSI scores compared to the 307 married respondents ( median $=1$, mean rank $=245.88$ vs median $=0$, mean rank $=209.50$ respectively) and compared to the 126 respondents living with their partner/de facto (median $=1$, mean rank $=139.71$ vs median $=0$, mean rank $=119.75$ respectively), Mann-Whitney $U=17,040, \quad Z=-2.95, \quad p=0.003$ and Mann-Whitney $U=7087, \quad Z=-2.25$, $p=0.024$ respectively. The 73 widowed/divorced/separated respondents had a median PGSI of 1 and did not differ significantly from any other group.

Significant differences in problem gambling severity were found between different household types, Kruskal-Wallis $\chi(5)=31.47, p<0.001$. The 38 respondents in one parent families with children (median $=5$ ) had significantly higher PGSI scores compared to the 215 respondents living as a couple with children (median $=5$, mean rank $=147.91$ vs median $=0$, mean rank $=123.30$ respectively) and the 186 respondents living as a couple with no children (median $=5$, mean rank $=148.32$ vs median $=0$, mean rank $=105.18$ respectively), Mann-Whitney $U=3290.5, Z=-2.03, p=0.043$ and Mann-Whitney $U=2173, Z=-4.16, p<0.001$ respectively. Furthermore, those living as a couple with no children had significantly lower PGSI scores compared to the 117 people living singly (median $=0$, mean rank $=138.40$ vs median $=1$, mean rank $=173.62$ respectively) and the 70 in group households (median $=0$, mean rank $=116.86$ vs median $=2$, mean rank $=159.42$ respectively), Mann-Whitney $U=8,351, \quad Z=-3.75, \quad p<0.001$ and Mann-Whitney $U=4,345.5, \quad Z=-4.48$, $p<0.001$ respectively. Finally, a significant difference was observed between those living as a couple with children compared to those living as a couple with no children. While these groups had the same medians (0), a non-parametric test demonstrated that the couple with children group (mean rank $=216.87$ ) had significantly higher PGSI scores compared to those living as a couple with no children (mean rank =182.66), Mann-Whitney $U=16,583.5, Z=-3.25, p=0.001$. The 13 respondents living in an 'other' arrangement did not differ significantly from any of the other groups.

Significant differences were found between those with different levels of education, Kruskal-Wallis $\chi(4)=9.84, p=0.043$. Specifically, the 93 respondents who did not complete high school (median $=0$ ) had a significantly lower level of problem gambling severity compared to the 133 with year 12 as their highest educational qualification (median $=0$, mean $\mathrm{rank}=103.44$ vs median $=1$, mean rank $=120.54$ respectively, Mann-Whitney $U=5248.5, Z=-2.07, p=0.039$ ), to the 146 respondents with an undergraduate qualification (median $=0$, mean rank $=106.99$ vs median $=1$, mean rank $=128.28$ respectively, Mann-Whitney $U=5579.5, Z=-2.48, p=0.013$ ) and to 
the 85 respondents with a postgraduate qualification (median $=0$, mean rank $=82.77$ vs median $=1$, mean rank $=96.86$ respectively, Mann-Whitney $U=3326.5, Z=-1.97$, $p=0.049$ ). Further, those with an undergraduate qualification (median $=1$, mean rank $=175.97$ ) had significantly higher PGSI scores compared to the 182 respondents with a trade or technical certificate or diploma (median $=0$, mean rank $=155.30$, MannWhitney $U=11,612.6, Z=-2.10, p=0.036$ ). No other group differences were significant.

Significant differences were found between those of differing work status, KruskalWallis $\chi(8)=24.53, p=0.002$. The 310 full-time respondents had significantly higher PGSI scores compared to the 54 self-employed respondents (median $=1$, mean rank $=188.20$ vs median $=0$, mean rank $=149.81$ respectively, Mann-Whitney $U=6604.5, Z=-2.60, p=0.009$ ), the 75 retired respondents (median $=1$, mean rank $=203.13$ vs median $=0$, mean rank $=151.14$ respectively, Mann-Whitney $U=8485.5, Z=-3.86, p<0.001)$ and the 24 respondents on sick or disability pensions (median $=1$, mean $\mathrm{rank}=170.30$ vs median $=0$, mean rank $=131.33$ respectively, Mann-Whitney $U=2852.0, Z=-2.00, p=0.046$ ). Further, the 21 full-time students had significantly higher PGSI scores compared to the self-employed (median $=1$, mean rank $=46.43$ vs median $=0$, mean rank $=34.72$ respectively, Mann-Whitney $U=390$, $Z=-2.25, p=0.025$ ), the retired (median $=1$, mean rank $=62.38$ vs median $=0$, mean rank $=44.61$ respectively Mann-Whitney $U=496, Z=-2.91, p=0.004$ ) and sick or disability pensioners (median $=1$, mean rank $=27.14$ vs median $=0$, mean rank $=19.38$ respectively, Mann-Whitney $U=165, Z=-2.11, p=0.035$ ). Finally, the 94 part-time workers had a median of 0 , as did the retired respondents, although the post hoc tests revealed a significant difference in ranked scores, with part-time workers having significantly higher scores (mean rank $=91.84$ vs mean rank $=76.43$ respectively), Mann-Whitney $U=2882.0, Z=-2.28, p=0.023$. The 25 unemployed respondents and 26 on full-time home duties did not differ significantly from any of the other groups and no other differences were significant. No significant relationship was found between household income and PGSI score (Spearman's rho $=0.013, p=0.757$ ).

\section{Sports Betting Behaviour}

Higher frequency of sports betting was related to higher PGSI scores (Spearman's rho $=0.48, p<0.001$ ), as was higher sports betting expenditure (Spearman's rho $=0.43$, $p<0.001$ ). The number of accounts respondents had with different sports betting agencies was not related to problem gambling severity (Spearman's rho $=0.03, p=0.431$ ).

In terms of betting behaviours (Table 1), significantly higher PGSI scores were found amongst those who placed a higher proportion of their bets via the telephone (as expected because live action bets can only be placed by telephone and in-person with licensed wagering operators in Australia), within an hour of match commencement or during the match, and on impulse before or during the match. Those who planned and researched a higher proportion of their bets in advance of the match had significantly lower PGSI scores than those who did not. Respondents who placed a higher proportion of their bets on the final outcome of the match, either before or during the game, had significantly lower PGSI scores than those who did not. Conversely, sports bettors who placed a higher proportion of their bets on key events (both for bets placed before or during the match) and on micro events during the match had significantly higher PGSI scores than those who did not.

Respondents who had engaged in more forms of gambling within the last 12 months (in addition to sports betting) were significantly more likely to have higher PGSI scores 
Table 1 Relationship between sports betting behaviours and PGSI scores

\begin{tabular}{|c|c|c|}
\hline Sports betting behaviours & $\begin{array}{l}\text { Spearman's } \\
\text { rho }\end{array}$ & $p$ \\
\hline \multicolumn{3}{|l|}{ Channel } \\
\hline$\%$ of bets placed via the internet & -0.07 & 0.076 \\
\hline$\%$ of bets placed via the telephone & 0.35 & $<0.001$ \\
\hline$\%$ of bets placed at a land-based venue & 0.03 & 0.523 \\
\hline \multicolumn{3}{|l|}{ Timing } \\
\hline$\%$ of bets placed before the day of the match & -0.06 & 0.120 \\
\hline$\%$ of bets placed on the day of the match & 0.01 & 0.777 \\
\hline$\%$ of bets placed within the hour immediately before the match starts & 0.25 & $<0.001$ \\
\hline$\%$ of bets placed during the match & 0.36 & $<0.001$ \\
\hline \multicolumn{3}{|l|}{ Planning } \\
\hline$\%$ of bets that are researched and planned in advance of the match & -0.25 & $<0.001$ \\
\hline$\%$ of bets that are placed on impulse before the start of the match & 0.18 & $<0.001$ \\
\hline$\%$ of bets that are placed on impulse during the match & 0.37 & $<0.001$ \\
\hline \multicolumn{3}{|l|}{ Type } \\
\hline$\%$ of bets placed on the final outcome of the match (placed before the match) & -0.33 & $<0.001$ \\
\hline $\begin{array}{l}\% \text { of bets placed on key events within the match (exotic bets), e.g., who will } \\
\text { score the first goal (placed before the match) }\end{array}$ & 0.33 & $<0.001$ \\
\hline$\%$ of bets placed on the final outcome of the match (placed during the match) & -0.41 & $<0.001$ \\
\hline $\begin{array}{l}\% \text { of bets placed on key events within the match (exotic bets), e.g., who will } \\
\text { score the first goal (placed during the match) }\end{array}$ & 0.31 & $<0.001$ \\
\hline $\begin{array}{l}\% \text { of bets placed on micro events within the match (exotic bets), e.g., the outcome } \\
\text { of the next ball in cricket or point in tennis (placed during the match) }\end{array}$ & 0.47 & $<0.001$ \\
\hline
\end{tabular}

The percentages for the items within each type of behaviour (channel, timing, planning and type) must sum to $100 \%$. Note that the items asked about the type of bets placed during the match were only asked of those who reported that they had placed bets during a match $(N=136)$

compared to those who had engaged in fewer other forms (Spearman's rho $=0.25$, $p<0.001)$.

\section{Normative Influences}

Those who watched both live sports at a sporting venue and televised sports more often had significantly higher PGSI scores than those who watched less often (Spearman's rho $=0.26$ and 0.22 respectively, both $p<0.001$ ). Respondents with significantly higher subjective norms (both for the family and friends subscales, as well as the total scale) had significantly higher PGSI scores than those with lower levels of subjective norms (Spearman's rho $=0.22,0.28$ and 0.26 respectively, all $p<0.001$ ).

\section{Discussion}

This study represents a first attempt to identify demographic, behavioural and normative risk factors for gambling problems amongst sports bettors. Identifying these risk factors is important, given the recent and expected future growth in sports betting, its increasing 
contribution to problem gambling, and the need for appropriate preventative, early intervention and treatment initiatives (Foley-Train 2014; Hing et al. 2014b, c).

Several demographic risk factors for problem gambling were identified amongst sports bettors. These included being male, younger and never married, and living either alone, in a one-parent family with children, or in a group household. These results confirm earlier research findings that young adult males are an at-risk group for problem gambling (Delfabbro 2012; Williams et al. 2012), and lend support to concerns about a growing culture of high risk sports betting amongst this cohort (Gordon et al. 2015; Sproston et al. 2015; Thomas et al. 2012). They also point to single marital status as a risk factor, perhaps because unpartnered sports bettors have fewer financial and family responsibilities that might help to restrain their gambling, they may be more likely to watch and bet on sports with their friends, and also because unmarried bettors may be more likely to frequent social settings such as bars where sports betting amongst young males often occurs (Gordon et al. 2015; Sproston et al. 2015). These findings indicate the need for public health interventions to minimise sports betting-related harm amongst young men. While public health strategies have focused most on EGM gambling, the growth and heavy promotion of sports betting suggest that the focus of these measures needs broadening and should especially target 18-35 year old males. The findings also point to the need for preventative measures, particularly targeting adolescent males and integrated into youth media and school educational programs, to avoid an escalation of gambling problems amongst young men once they reach the legal age for sports betting. The high exposure of adolescents to advertisements and promotions for sports betting (Hing et al. 2014d; Sproston et al. 2015) suggests that such measures would be prudent.

The risk of problem gambling was also elevated amongst sports bettors with higher levels of education and amongst those working or studying full-time. This aligns with the previously identified profile of Internet gamblers as more likely to be better educated, studying or working full-time in managerial or professional occupations, and earning above average salaries which provides them with more disposable income (Gainsbury 2012). Our demographic profile of higher risk sports bettors also aligns with the target market of most Australian wagering operators who position sports betting as an activity engaged in by young, single, upwardly mobile, professional, tech-savvy young men (Hing et al. 2014c; Milner et al. 2013; Sproston et al. 2015). For example, key emotions and meanings in Australian wagering advertising have been found to include sophistication, excitement, power, mateship, wealth, a glamorous high stakes lifestyle and personal success, with ease of access through mobile betting technologies also emphasised (Lamont et al. 2015; Sproston et al. 2015). Social marketing messages targeting this same demographic profile are needed to offset the persuasive appeal of the proliferation of these sports betting messages now aimed at this group through a wide range of digital and traditional media. While sports betting advertisements currently include a mandated 'gamble responsibly' message in Australia, these are easily missed due to their typical lack of prominence (Lamont et al. 2015; Sproston et al. 2015). Further, website and social media advertisements for sports betting do not commonly display responsible gambling messages (Gainsbury et al. 2015).

Several risk factors were also identified in terms of sports betting and other gambling behaviours. Sports bettors who engaged in more forms of gambling had higher problem gambling severity, consistent with previous findings that extent of gambling involvement is a key predictor of problem gambling and one that explains much of the variation in problem gambling rates between Internet and non-Internet gamblers (Gainsbury et al. 2015; Wood and Williams 2011). Not surprisingly, higher levels of sports betting 
frequency and expenditure were also associated with elevated PGSI scores, in alignment with previous research based on wagering operator data (LaBrie and Shaffer 2011; LaPlante et al. 2014).

Higher problem gambling severity was also associated with a less planned approach to betting, as reflected in a higher proportion of betting: by telephone (as live action bets can only be placed by telephone and in-person with licensed wagering operators in Australia); on key events and micro events; immediately before match commencement or during the match; and on impulse before or during the match. These findings provide convergent evidence that impulse betting, and the provision of betting options that enable this, pose substantial risks for some bettors. Live action betting on contingencies during play has been identified as a risk factor in previous research (Braverman et al. 2013; Gray et al. 2012; LaPlante et al. 2008, 2014; Nelson et al. 2008), while an experimental study found that problem gamblers were particularly tempted by live action micro-bets (Hing et al. 2014c). In Australia, live action betting over the Internet is prohibited with licensed bookmakers, in recognition that it provides opportunities for continuous, rapid and repetitive betting (DBCDE 2013), which may pose particular risks for gamblers with impulse control difficulties. However, live action bets can still be made by telephone, in retail outlets and through offshore operators illegally providing betting services to Australians. Further, some Australian licensed bookmakers are now offering a click-to-call option which allows bettors to place live action bets through computer or mobile phone without needing to speak to a telephone operator. The availability of this new option and the ease with which Australians can bet with offshore operators means that current bans on online live action betting are easy to avoid. Thus, if sports betting-related harm is to be minimised, it is imperative that responsible gambling measures of online bookmakers, such as facilities to set bet and deposit limits and to self-exclude, are rigorous, efficacious and well promoted to bettors. Improving sports bettors' understanding of betting odds might also be a worthwhile intervention and component of treatment.

Normative factors were also associated with higher problem gambling severity. Those who more frequently watched both live and televised sporting events (where sports betting is heavily promoted) were more likely to have higher PGSI scores. While this result may indicate that greater exposure to sports betting promotions increases the risk of problem gambling, an alternative explanation is that higher risk sports bettors watch more sporting events. In either case, exposure to sports betting marketing is a risk factor for gambling problems amongst sports bettors, suggesting that reducing the possible effects of this exposure, either through curtailing this marketing or by educating consumers about its normalising effects, is desirable to prevent and reduce sports betting problems.

Normative influences from significant others were also associated with elevated problem gambling risk. This finding may reflect that high risk sports bettors experience family and peer pressure to sports bet, or that they gravitate towards friendship groups which are supportive of this activity. Evidence for both these effects amongst young Australian male sports bettors has been found (Gordon et al. 2015; Thomas et al. 2012). These apparent normative effects from both the media and significant others suggest that counter messages, such as education programs in schools and in public health campaigns, are needed to raise awareness of the risks associated with sports betting and to temper emerging social norms that betting is an integral and harmless part of sport watching and of socialising with peers.

The current study used a purposive sample, so the problem gambling rates found should not be considered representative. Nevertheless, other research has indicated the growing association of problem gambling with sports betting (Hing et al. 2014b; Williams et al. 
2012). While preventative and early intervention measures, as suggested above, may help to reduce and minimise sports betting-related harm, treatment services are needed for severe cases. To date, gambling treatment services have been utilised mainly by people with EGM-related problems. However, these services may need to be better equipped to treat sports bettors by ensuring they have therapists who can relate to young males, understand the features of sports betting that can contribute to loss of control, and use treatments that help clients to resist sports betting urges. Online treatment services may better suit online bettors, but low uptake of professional treatment amongst problem gamblers (Cunningham 2005) suggests that development of a range of self-help resources for problem sports bettors is needed and especially targeted to the profile of higher risk sports bettors revealed in this study.

Other limitations of this study include its cross-sectional design which prevents determination of causality, and its collection of only self-report data that may be subject to social desirability, recall and other biases. Further research is needed with representative samples, in other jurisdictions and using prospective designs to confirm the current results. Future studies could also examine other types of risk factors, including psychological, social and cultural variables. Research into the role of sports betting inducements, such as sign-up bonuses, 'free' bets, money-back guarantees and other prolific offers, would also help to identify their influence on individuals' commencement, continuation and intensification of sports betting.

\section{Conclusion}

Understanding risk factors for problem gambling amongst sports bettors is an increasingly important area of research, given the exponential growth of sports betting in many countries, its heavy promotion and its successful targeting of young adult males. This study found that vulnerable sports bettors for higher risk gambling are those who are young, male, single, educated, and employed or a full-time student. Further, risk increases with greater frequency and expenditure on sports betting and with more impulsive responses to betting opportunities, particularly live action betting. Normative influences from media advertising and from significant others were also associated with greater gambling risk. This study is the first to identify demographic, behavioural and normative risk factors amongst sports bettors and its results can inform a suite of prevention, protection and treatment initiatives that can help to limit harm from this gambling form.

Acknowledgments This study was funded by a Responsible Gambling Research Grant from the Queensland Department of Justice and Attorney General. The authors would also like to thank Dr Elian Fink who assisted with data cleaning and some preliminary analyses.

\section{Compliance with Ethical Standards}

Conflicts of interest The authors have no conflicts of interest to declare in relation to this paper.

\section{References}

Australian Communication and Media Authority (ACMA). (2013). Betting odds and advertising for betting agencies during sports broadcasts: Community research. Canberra: ACMA.

Blaszczynski, A., \& Hunt, C. (2011). Online sports betting has created new generation of problem gamblers. Sydney: The University of Sydney, 2011. http://sydney.edu.au/news/84.html?newsstoryid=6545. 
Borland, J., \& McDonald, R. (2003). Demand for sport. Oxford Review of Economic Policy, 19, 478-502.

Braverman, J., LaBrie, R. A., \& Shaffer, H. J. (2011). A taxometric analysis of actual internet sports gambling behavior. Psychological Assessment, 23(1), 234.

Braverman, J., LaPlante, D. A., Nelson, S. E., \& Shaffer, H. J. (2013). Using cross-game behavioral markers for early identification of high-risk internet gamblers. Psychology of Addictive Behaviors, 27(3), 868.

Broda, A., LaPlante, D. A., Nelson, S. E., LaBrie, R. A., Bosworth, L. B., \& Shaffer, H. J. (2008). Virtual harm reduction efforts for internet gambling: Effects of deposit limits on actual Internet sports gambling behavior. Harm Reduction Journal, 5(1), 27.

Cunningham, J. A. (2005). Little use of treatment among problem gamblers. Psychiatric Services, 56, 1024-1025.

Delfabbro, P. H. (2012). Australasian gambling review (5th ed.). Adelaide: Independent Gambling Authority.

Department of Broadband Communications and the Digital Economy (DBCDE). (2013). Final report 2012: Review of the interactive gambling act 2001. Canberra: DBCDE.

Ferris, J., \& Wynne, H. (2001). The Canadian problem gambling index: Final report. Ottawa: Canadian Centre on Substance Abuse.

Foley-Train, J. (2014). Sports betting: Commercial and integrity issues. Report prepared for the Association of British Bookmakers, European Gaming and Betting Association, European Sport Security Association and Remote Gambling Association. Retrieved January 21, 2015 from http://www.egba.eu/ media/Sports-Betting-Report-FINAL.pdf.

Gainsbury, S. (2012). Internet gambling: Current research findings and implications. New York: Springer.

Gainsbury, S. M., Delfabbro, P., King, D. L., \& Hing, N. (2015a). An exploratory study of gambling operators' use of social media and the latent messages conveyed. Journal of Gambling Studies. doi:10. 1007/s10899-015-9525-2.

Gainsbury, S., Russell, A., Blaszczynski, A., \& Hing, N. (2015). Greater involvement and diversity of Internet gambling as a risk factor for problem gambling. European Journal of Public Health. http://dx. doi.org/10.1093/eurpub/ckv006.

Gainsbury, S., Russell, A., Hing, N., Wood, R., Lubman, D., \& Blaszczynski, A. (2013). How the internet is changing gambling: Findings from an Australian prevalence survey. Journal of Gambling Studies, 31(1), 1-15. doi:10.1007/s10899-013-9404-7.

Gainsbury, S., Wood, R., Russell, A., Hing, N., \& Blaszczynski, A. (2012). A digital revolution: Comparison of demographic profiles, attitudes and gambling behaviour of Internet and non-internet gamblers. Computers in Human Behaviour, 28(12), 1388-1398.

Global Betting and Gaming Consultants (GBGC). (2013). Global sports betting: The state of play. Retrieved January 21, 2015 from http://www.gbgc.com/global-sports-betting-the-state-of-play/.

Gordon, R., Gurrieri, L., \& Chapman, M. (2015). Broadening an understanding of problem gambling: The lifestyle consumption community of sports betting. Journal of Business Research. doi:10.1016/j. jbusres.2015.03.016.

Gray, H. M., LaPlante, D. A., \& Shaffer, H. J. (2012). Behavioral characteristics of internet gamblers who trigger corporate responsible gambling interventions. Psychology of Addictive Behaviors, 26(3), 527.

Hammervold, R., \& Solberg, H. (2006). TV sports programs: Who is willing to pay to watch? Journal of Media Economics, 19, 147-162.

Hing, N., Cherney, L., Gainsbury, S., Lubman, D., Wood, R., \& Blaszczynski, A. (2014a). Maintaining and losing control during Internet gambling: A qualitative study of gamblers' experiences. New Media and Society. doi:10.1177/1461444814521140.

Hing, N., Gainsbury, S., Blaszczynski, A., Wood, R., Lubman, D., \& Russell, A. (2014b). Interactive gambling. Melbourne: Gambling Research Australia.

Hing, N., Lamont, M., Vitartas, P., \& Fink, E. (2015a). Sports-embedded gambling promotions: A study of exposure, sports betting intention and problem gambling amongst adults. International Journal of Mental Health and Addiction, 13(1), 115-135. doi:10.1007/s11469-014-9519-9.

Hing, N., Russell, A., Tolchard, B., \& Nower, L. (2015b). Risk factors for gambling problems: An analysis by gender. Journal of Gambling Studies. doi:10.1007/s10899-015-9548-8.

Hing, N., Vitartas, P., \& Lamont, M. (2014c). Promotion of gambling and live betting odds during televised sport: Influences on gambling participation and problem gambling. Brisbane: Report prepared for the Queensland Department of Justice and Attorney-General. doi:10.13140/2.1.1643.8087.

Hing, N., Vitartas, P., Lamont, M., \& Fink, E. (2014d). Adolescent exposure to gambling promotions during televised sport: An exploratory study of links with gambling intentions. International Gambling Studies, 14(3), 374-393. doi:10.1080/14459795.2014.902489. 
Humphreys, B. R., \& Pérez, L. (2012). Who bets on sports? Characteristics of sports bettors and the consequences of expanding sports betting opportunities. Estudios de economía aplicada, 30(2), 579-597.

Johansson, A., Grant, J. E., Kim, S. W., Odlaug, B. L., \& Götestam, K. G. (2009). Risk factors for problematic gambling: A critical literature review. Journal of Gambling Studies, 25(1), 67-92.

Joint Select Committee on Gambling Reform (JSCGR). (2013). The advertising and promotion of gambling services in sport. Canberra: Commonwealth of Australia.

Kessler, R. C., Hwang, I., LaBrie, R., Petukhova, M., Sampson, N. A., Winters, K. C., \& Shaffer, H. J. (2008). DSM-IV pathological gambling in the national comorbidity survey replication. Psychological Medicine, 38(09), 1351-1360.

LaBrie, R. A., LaPlante, D. A., Nelson, S. E., Schumann, A., \& Shaffer, H. J. (2007). Assessing the playing field: A prospective longitudinal study of internet sports gambling behavior. Journal of Gambling Studies, 23(3), 347-362.

LaBrie, R., \& Shaffer, H. J. (2011). Identifying behavioral markers of disordered internet sports gambling. Addiction Research and Theory, 19(1), 56-65.

Lamont, M., Hing, N., \& Gainsbury, S. (2011). Gambling on sport sponsorship: A conceptual framework for research and regulatory review. Sport Management Review, 14, 246-257.

Lamont, M., Hing, N., \& Vitartas, P. (2015). Affective responses to gambling promotions during televised sport: A qualitative analysis. Sport Management Review. doi:10.1016/j.smr.2015.06.002.

LaPlante, D. A., Nelson, S. E., \& Gray, H. M. (2014). Breadth and depth involvement: Understanding internet gambling involvement and its relationship to gambling problems. Psychology of Addictive Behaviors, 28(2), 396-403.

LaPlante, D. A., Schumann, A., LaBrie, R. A., \& Shaffer, H. J. (2008). Population trends in Internet sports gambling. Computers in Human Behavior, 24(5), 2399-2414.

McMullan, J. L. (2011). Submission to the joint select committee on gambling reform inquiry into interactive gambling. Retrieved January 30, 2015 from http://www.aph.gov.au/DocumentStore.ashx?id= 40e3c180-5666-4084-92c6-0e51b62d7d0b.

Milner, L., Hing, N., Vitartas, P., \& Lamont, M. (2013). An exploratory study of embedded gambling promotion in Australian football television broadcasts. Communication, Politics and Culture, 46, 177-198.

Moore, S. M., \& Ohtsuka, K. (1997). Gambling activities of young Australians: Developing a model of behavior. Journal of Gambling Studies, 13, 207-236.

Nelson, S. E., LaPlante, D. A., Peller, A. J., Schumann, A., LaBrie, R. A., \& Shaffer, H. J. (2008). Real limits in the virtual world: Self-limiting behavior of Internet gamblers. Journal of Gambling Studies, 24(4), 463-477.

Philander, K. S. (2014). Identifying high-risk online gamblers: A comparison of data mining procedures. International Gambling Studies, 14(1), 53-63.

Problem Gambling Research and Treatment Centre. (2011). Guidelines for screening, assessment and treatment in problem gambling. Clayton: Monash University.

PWC (2011). Changing the game: Outlook for the global sports market to 2015. Retrieved January 21, 2015 from http://www.pwc.com/en_gx/gx/hospitality-leisure/pdf/changing-the-game-outlook-for-the-global-sportsmarket-to-2015.pdf.

Queensland Government Statistician's Office (QGSO). (2014). Australian gambling statistics (30th ed.). Brisbane: Queensland Government.

Sproston, K., Hanley, C., Brook, K., Hing, N., \& Gainsbury, S. (2015). Marketing of sports betting and racing. Melbourne: Gambling Research Australia.

Sproston, K., Hing, N., \& Palankay, C. (2012). Prevalence of gambling and problem gambling in New South Wales. Sydney: NSW Office of Liquor, Gaming and Racing.

Thomas, S. L., Lewis, S., McLeod, C., \& Haycock, J. (2012). They are working every angle. A qualitative study of Australian adults' attitudes towards, and interactions with, gambling industry marketing strategies. International Gambling Studies, 12(1), 111-127.

University of Sydney Gambling Treatment Clinic. (2011). Online sports betting has created new generation of problem gamblers. Media release. Retrieved December 9, 2013 from http://sydney.edu.au/news/84. html?newsstoryid=6545.

Wardle, H. \& Seabury, C. (2012). Gambling behaviour. In Health survey for England-2012 (Chapter 7). Retrieved January 21, 2015 from http://www.hscic.gov.uk/catalogue/PUB13218/HSE2012-Ch7Gambling-behaviour.pdf.

Weibe, J. (2008). Internet gambling: Strategies to recruit and retain customers. Guelph: Ontario Problem Gambling Research Centre. 
Welte, J. W., Barnes, G. M., Wieczorek, W. F., Tidwell, M. C. O., \& Parker, J. C. (2004). Risk factors for pathological gambling. Addictive Behaviors, 29(2), 323-335.

Williams, R. J., Volberg, R. A., \& Stevens, R. M. (2012a). The population prevalence of problem gambling: Methodological influences, standardized rates, jurisdictional differences, and worldwide trends. Ontario: Problem Gambling Research Centre.

Williams, R. J., West, B. L., \& Simpson, R. I. (2012b). Prevention of problem gambling: A comprehensive review of the evidence and identified best practices. Guelph, ON: Ontario Problem Gambling Research Centre.

Wood, R. T., \& Williams, R. J. (2011). A comparative profile of the internet gambler: Demographic characteristics, game-play patterns, and problem gambling status. New Media and Society, 13(7), 1123-1141.

Xuan, Z., \& Shaffer, H. (2009). How do gamblers end gambling: Longitudinal analysis of internet gambling behaviors prior to account closure due to gambling related problems. Journal of Gambling Studies, 25, 239-252. 